\title{
Approximate pexiderized gamma-beta type functions
}

\section{Young Whan Lee*}

"Correspondence: ywlee@dju.kr Department of Computer Hacking and Information Security College of Natural Science, Daejeon University, Daejeon, 300-716, Republic of Korea

\begin{abstract}
We show that every unbounded approximate pexiderized gamma-beta type function has a gamma-beta type. That is, we obtain the superstability of the pexiderized gamma-beta type functional equation

$$
\beta(x, y) f(x+y)=g(x) h(y)
$$

and also investigate the superstability as the following form:

$$
\left|\frac{\beta(x, y) f(x+y)}{g(x) h(y)}-1\right| \leq \varphi(x, y)
$$

MSC: 39B72; 38B22; 39B82

Keywords: functional equation; stability; superstability; gamma and beta functional equation; Cauchy functional equation; exponential functional equation
\end{abstract}

\section{Introduction}

In 1940, Ulam gave a wide ranging talk in the Mathematical Club of the University of Wisconsin in which he discussed a number of important unsolved problems (ref. [1]). Among those there was a question concerning the stability of homomorphisms: Let $G_{1}$ be a group and let $G_{2}$ be a metric group with a metric $d(\cdot, \cdot)$. Given $\epsilon>0$, does there exist a $\delta>0$ such that if a mapping $h: G_{1} \rightarrow G_{2}$ satisfies the inequality $d(h(x y), h(x) h(y))<\delta$ for all $x, y \in G_{1}$, then there exists a homomorphism $H: G_{1} \rightarrow G_{2}$ with $d(h(x), H(x))<\epsilon$ for all $x \in G_{1}$ ? In the next year, Hyers [2] answered the question of Ulam for the case where $G_{1}$ and $G_{2}$ are Banach spaces. Furthermore, the result of Hyers was generalized by Rassias [3]. Since then, the stability problems of various functional equations have been investigated by many authors (ref. [4]).

Baker, Lawrence and Zorzitto [5] proved the Hyers-Ulam stability of the Cauchy exponential equation $f(x+y)=f(x) f(y)$. That is, if the Cauchy difference $f(x+y)-f(x) f(y)$ of a real-valued function $f$ defined on a real vector space is bounded for all $x, y$, then $f$ is either bounded or exponential. Their result was generalized by Baker [6]: Let $S$ be a semi-group and let $f: S \rightarrow E$ be a mapping where $E$ is a normed algebra in which the norm is multiplicative. If $f$ satisfies the functional inequality $\|f(x y)-f(x) f(y)\| \leq \delta$ for all $x, y \in S$, then $f$ is either bounded or multiplicative. That is, every unbounded approximate multiplica- 
tive function is multiplicative. Such a phenomenon for functional equations is called the superstability.

The author [7] proved superstability of the pexiderized multiplicative functional equation

$$
f(x+y)=g(x) h(x)
$$

and the author and Kim [8] also obtained superstability of the gamma-beta type functional equation

$$
\beta(x, y) f(x+y)=f(x) f(y),
$$

where $\beta(x, y)$ is a beta-type function.

In this paper, we generalize it to the pexiderized gamma-beta type functional equation

$$
\beta(x, y) f(x+y)=g(x) h(y) .
$$

And then we prove the superstability of this equation and obtain the superstability in the sense of Ger [9].

\section{Definitions and solutions}

Throughout this paper, we denote by $D$ an additive subset (that is, $x+y \in D$ for all $x, y \in D$ ) of $R$ containing all positive integers $Z^{+}$.

Definition 1 Let a function $\beta: D \times D \rightarrow R-\{0\}$ satisfy the following conditions (a) $\sim(\mathrm{e})$ :

(a) $\beta(x, y)=\beta(y, x)(x, y \in D)$,

(b) $|\beta(n, m)| \leq 1\left(n, m \in Z^{+}\right)$,

(c) $\frac{\beta(x, y) \beta(z, x+y)}{\beta(x, y+z) \beta(y, z)}=1(x, y \in D)$,

(d) $\lim _{n \rightarrow \infty} \prod_{i=1}^{n-1}|\beta(i m, m)|=0\left(m \in Z^{+}\right)$,

(e) $|\beta(x, n)|<\infty\left(n \in Z^{+}\right.$and fixed $\left.x \in D\right)$.

Then we call $\beta$ a beta-type function.

Definition 2 Let a function $\varphi: D \rightarrow[0, \infty)$ and a beta-type function $\beta: D \times D \rightarrow R-\{0\}$ be given. If a function $f: D \rightarrow R$ satisfies that

$$
|\beta(x, y) f(x+y)-f(x) f(y)| \leq \varphi(x, y)
$$

for all $(x, y) \in D \times D$, then we call $f$ a $\{\varphi, \beta\}$-approximate gamma-beta type function. In the case of $\varphi=0$, we call $f$ a gamma-beta type function.

Definition 3 Let a function $\varphi: D \rightarrow[0, \infty)$ and a beta-type function $\beta: D \times D \rightarrow R-\{0\}$ be given. If a function $f: D \rightarrow R$ satisfies that

$$
|\beta(x, y) f(x+y)-g(x) h(y)| \leq \varphi(x, y)
$$

for all $(x, y) \in D \times D$ and for some functions $g, h: D \rightarrow R$, then we call $f$ a $\{\varphi, \beta\}$ approximate pexiderized gamma-beta type function. In the case of $\varphi=0$, we call $f$ a pexiderized gamma-beta type function. 


\section{Examples and solutions}

If $f, g, h: R^{+} \rightarrow R^{+}$are functions satisfying equation (1.1) and $\beta(x, y)=\frac{1}{a^{x y}}(a>1)$, then $\beta$ is a beta-type function and $f(x)=a^{\frac{x^{2}}{2}+3}, g(x)=a^{\frac{x^{2}}{2}+2}, h(x)=a^{\frac{x^{2}}{2}+1}$ are solutions of it.

Now, we consider the gamma and the beta functions. Note that the beta function $B(x, y)$ is defined by

$$
B(x, y)=\int_{0}^{1} t^{x-1}(1-t)^{y-1} d t \quad(x>0, y>0)
$$

and the gamma function is defined by

$$
\Gamma(x)=\int_{0}^{\infty} e^{-t} t^{x-1} d t \quad(x>0) .
$$

It is well known that $B$ and $\Gamma$ satisfy the gamma-beta functional equation

$$
B(x, y) \Gamma(x+y)=\Gamma(x) \Gamma(y)
$$

for all $x, y \in(0, \infty)$. Also, $B(x, y)=B(y, x)$ and

$$
B(n, m)=\frac{\Gamma(n) \Gamma(m)}{\Gamma(n+m)}=\frac{(n-1) !(m-1) !}{(n+m-1) !}<1
$$

for all $x, y \in(0, \infty)$ and nonnegative integers $n, m$. By (2.1), we have

$$
\begin{aligned}
\prod_{i=1}^{n-1} B(i m, m) & =\frac{\Gamma(m) \Gamma(m)}{\Gamma(2 m)} \cdot \frac{\Gamma(2 m) \Gamma(m)}{\Gamma(3 m)} \cdots \frac{\Gamma((n-1) m) \Gamma(m)}{\Gamma(n m)} \\
& =\frac{\Gamma(m)^{n}}{\Gamma(n m)} \\
& =\frac{[(m-1) !]^{n}}{(n m-1) !} \rightarrow 0
\end{aligned}
$$

as $n \rightarrow \infty$ and

$$
\frac{B(x, y) B(z, x+y)}{B(x, y+z) B(y, z)}=1
$$

for all $x, y, z \in(0, \infty)$. Also, for all $x \in(0, \infty)$ and $n \in Z^{+}$,

$$
B(x, n)=\frac{\Gamma(n) \Gamma(x)}{\Gamma(x+n)}=\frac{(n-1) !}{(x+n-1)(x+n-2) \cdots(x+1) x}<\frac{1}{x} .
$$

Thus, $B:(0, \infty) \times(0, \infty) \rightarrow(0, \infty)$ is a beta-type function and $\Gamma$ is a gamma-beta type function.

If $\beta(x, y)$ is the beta function and

$$
f(x)=6 a^{x+1} \Gamma(x), \quad g(x)=3 a^{x} \Gamma(x), \quad h(x)=2 a^{x+1} \Gamma(x),
$$

then $f, g, h$ are the solutions of equation (1.1). 


\section{Superstability of a gamma-beta type functional equation}

The following Theorem 1 with $\phi(x)=\delta$ states that every unbounded approximate pexiderized gamma-beta type function is a gamma-beta type function.

Theorem 1 Let a function $\phi: D \rightarrow[0, \infty)$ be given and let $\varphi(x, y)=\min \{\phi(x), \phi(y)\}$. Suppose that $\beta: D \times D \rightarrow R-\{0\}$ is a beta-type function and $f, g, h: D \rightarrow R$ are functions such that $f$ is a $\{\varphi, \beta\}$-approximate gamma-beta type function, $f(s)=g(s)$ for some $s \in D$ and

$$
|\beta(x, y) f(x+y)-g(x) h(y)| \leq \varphi(x, y)
$$

for all $(x, y) \in D \times D$.

(a) If $h$ is unbounded, then $f$ and $g$ are unbounded gamma-beta type functions.

(b) If $|g(m)| \geq \max \{2,(8 \phi(s)+4 \phi(m)) /|h(m)||g(s)|\}$ for some positive integer $m$, then $f$ and $g$ are unbounded gamma-beta type functions.

Proof (a) Suppose that $h$ is unbounded. Since $f$ is a $\{\varphi, \beta\}$-approximate gamma-beta type function,

$$
\begin{aligned}
|h(x)-f(x)| & =\frac{1}{|g(s)|}|g(s) h(x)-f(s) f(x)| \\
& \leq \frac{|\beta(x, s) f(x+s)-f(x) f(s)|+|\beta(x, s) f(x+s)-h(x) g(s)|}{|g(s)|} \\
& \leq \frac{2 \phi(s)}{|g(s)|}
\end{aligned}
$$

for all $x \in D$. Also, since

$$
\begin{aligned}
|g(x) h(y)-g(y) h(x)| & \leq|g(x) h(y)-\beta(x, y) f(x+y)|+|\beta(y, x) f(x+y)-g(y) h(x)| \\
& \leq 2 \phi(y)
\end{aligned}
$$

we have

$$
|g(x)| \leq|h(x)|\left|\frac{g(y)+2 \phi(y)}{h(y)}\right|
$$

and

$$
|h(x)| \leq|g(x)| \frac{h(y)+2 \phi(y)}{g(y)} \mid
$$

for all $x \in D$ and for fixed $y \in D$. Thus, $f$ and $g$ are unbounded. By the unboundedness of $h$, we can choose a sequence $\left\{y_{n}\right\}$ in $Z^{+}$such that $\left|h\left(y_{n}\right)\right| \rightarrow \infty$ as $n \rightarrow \infty$. By the conditions (a), (c) and (e) of the beta-type function $\beta$ and (3.2), we have

$$
\begin{aligned}
& \left|h\left(y_{n}\right)\right||\beta(x, y) g(x+y)-g(x) g(y)| \\
& \quad \leq|\beta(x, y)|\left|h\left(y_{n}\right) g(x+y)-\beta\left(x+y, y_{n}\right) f\left(x+y+y_{n}\right)\right| \\
& \quad+\left|\beta\left(y, y_{n}\right)\right|\left|\beta\left(x, y+y_{n}\right) f\left(x+y+y_{n}\right)-g(x) h\left(y+y_{n}\right)\right|
\end{aligned}
$$




$$
\begin{gathered}
+\left|\beta\left(y, y_{n}\right)\right|\left|h\left(y+y_{n}\right)-f\left(y+y_{n}\right)\right||g(x)| \\
+|g(x)|\left|\beta\left(y, y_{n}\right) f\left(y+y_{n}\right)-g(y) h\left(y_{n}\right)\right| \\
\leq|\beta(x, y)| \phi(x+y)+\left|\beta\left(y, y_{n}\right)\right| \phi(x) \\
+|g(x)|\left|\beta\left(y, y_{n}\right)\right| \frac{2 \phi(s)}{|g(s)|}+|g(x)| \phi(y)<\infty
\end{gathered}
$$

for all sufficiently large $y_{n}$ and $(x, y) \in D \times D$. It follows from (3.3) by dividing $\left|h\left(y_{n}\right)\right|$ that

$$
\beta(x, y) g(x, y)=g(x) g(y)
$$

for all $(x, y) \in D \times D$. Also, by letting $f=g=h$ in (3.3) and using the property of an approximate gamma-beta type function, we have

$$
\beta(x, y) f(x, y)=f(x) f(y)
$$

for all $(x, y) \in D \times D$.

(b) If we replace $x$ by $m$ and also $y$ by $m$ in (3.1), respectively, we get

$$
|\beta(m, m) f(2 m)-g(m) h(m)| \leq \phi(m) .
$$

Note that $|f(x)-h(x)| \leq 2 \phi(s) /|g(s)|$ from the proof of (a). An induction argument implies that for all $n \geq 2$,

$$
\begin{aligned}
& \left|f(n m) \prod_{i=1}^{n-1} \beta(i m, m)-g(m)^{n-1} h(m)\right| \\
& \leq \phi(m) \prod_{i=1}^{n-2}|\beta(i m, m)| \\
& \quad+|g(m)|\left(\frac{2 \phi(s)}{|g(s)|} \prod_{i=1}^{n-2}|\beta(i m, m)|+\phi(m) \prod_{i=1}^{n-3}|\beta(i m, m)|\right) \\
& \quad+|g(m)|^{2}\left(\frac{2 \phi(s)}{|g(s)|} \prod_{i=1}^{n-3}|\beta(i m, m)|+\phi(m) \prod_{i=1}^{n-4}|\beta(i m, m)|\right) \\
& \quad+\cdots+|g(m)|^{n-2}\left(\frac{2 \phi(s)}{|g(s)|}|\beta(m, m)|+\phi(m)\right) .
\end{aligned}
$$

To prove the inequality (3.4) by the induction, suppose that the inequality (3.4) holds for $k=n \geq 2$. Let $k=n+1$. Then we have

$$
\begin{aligned}
& \left|f((n+1) m) \prod_{i=1}^{n} \beta(i m, m)-g(m)^{n} h(m)\right| \\
& \leq|\beta(n m, m) f((n+1) m)-g(m) h(n m)| \prod_{i=1}^{n-1}|\beta(i m, m)| \\
& \quad+|g(m)||h(n m)-f(n m)| \prod_{i=1}^{n-1}|\beta(i m, m)|
\end{aligned}
$$




$$
\begin{aligned}
& +|g(m)| f(n m) \prod_{i=1}^{n-1} \beta(i m, m)-g(m)^{n-1} h(m) \mid \\
\leq & \phi(m) \prod_{i=1}^{n-1}|\beta(i m, m)|+|g(m)| \frac{2 \phi(s)}{|g(s)|} \prod_{i=1}^{n-1}|\beta(i m, m)| \\
& +|g(m)|\left|f(n m) \prod_{i=1}^{n-1} \beta(i m, m)-g(m)^{n-1} h(m)\right|
\end{aligned}
$$

for all $n \geq 2$. And thus we get

$$
\begin{aligned}
&\left|f((n+1) m) \prod_{i=1}^{n} \beta(i m, m)-g(m)^{n} h(m)\right| \\
& \leq \phi(m) \prod_{i=1}^{n-1}|\beta(i m, m)| \\
&+|g(m)|\left(\frac{2 \phi(s)}{|g(s)|} \prod_{i=1}^{n-1}|\beta(i m, m)|+\phi(m) \prod_{i=1}^{n-2}|\beta(i m, m)|\right) \\
&+|g(m)|^{2}\left(\frac{2 \phi(s)}{|g(s)|} \prod_{i=1}^{n-2}|\beta(i m, m)|+\phi(m) \prod_{i=1}^{n-3}|\beta(i m, m)|\right) \\
&+\cdots+|g(m)|^{n-1}\left(\frac{2 \phi(s)}{|g(s)|}|\beta(m, m)|+\phi(m)\right)
\end{aligned}
$$

for all $n \geq 2$. By the induction, the inequality (3.4) holds for all $n \in Z+$. Note that

$$
\prod_{i=1}^{n-1} \beta(i m, m)<\infty, \quad \text { and } \quad\left|g(m)^{n-1}\right| \rightarrow \infty \quad \text { as } n \rightarrow \infty .
$$

By dividing $g(m)^{n-1} h(m)$ by (3.4), we get

$$
\begin{aligned}
\left|\frac{f(n m) \prod_{i=1}^{n-1} \beta(i m, m)}{g(m)^{n-1} h(m)}-1\right| & \\
\leq & \frac{1}{g(m)^{n-1} h(m)}\left(\phi(m)+\left(\frac{2 \phi(s)}{|g(s)|}+\phi(m)\right)|g(m)|\right. \\
& \left.+|g(m)|^{2}\left(\frac{2 \phi(s)}{|g(s)|}+\phi(m)\right)+\cdots+|g(m)|^{n-2}\left(\frac{2 \phi(s)}{|g(s)|}+\phi(m)\right)\right) \\
\leq & \frac{2 \phi(s)+\phi(m)}{|h(m)||g(m)||g(s)|}\left(\frac{1}{|g(m)|^{m-2}}+\frac{1}{|g(m)|^{m-3}}+\cdots+\frac{1}{|g(m)|}+1\right) \\
\leq & \frac{2 \phi(s)+\phi(m)}{|h(m)||g(m)||g(s)|}\left(1+\frac{1}{2}+\frac{1}{2^{2}}+\frac{1}{2^{3}} \cdots\right) \\
\leq & 2 \frac{2 \phi(s)+\phi(m)}{|h(m)||g(m)||g(s)|} \\
\leq & \frac{1}{2}
\end{aligned}
$$


for all positive integer $n$. Thus, we can easily show that

$$
|f(n m)| \rightarrow \infty \quad \text { as } n \rightarrow \infty
$$

Thus, $f$ is unbounded and so $h$ is unbounded. By (a), we complete the proof.

Corollary 1 Let $\delta>0$ be given and $\beta(x, y)$ be a beta-type function on $(0, \infty)$. Suppose that $f$ is a function from $(0, \infty)$ into $(0, \infty)$ with $f(m) \geq \max \left\{2,(12 \delta)^{1 / 3}\right\}$ for some positive integer $m$ such that

$$
|\beta(x, y) f(x+y)-f(x) f(y)| \leq \delta
$$

for all $x, y \in(0, \infty)$. Then

$$
\beta(x, y) f(x+y)=f(x) f(y)
$$

for all $x, y \in(0, \infty)$.

Proof By Theorem 1 with $\phi(x)=\delta$ and $s=m$, we complete the proof.

Corollary 2 Let $\delta>0$ be given. Suppose that $h, g:(0, \infty) \rightarrow(0, \infty)$ are functions with $g(1)=$ $1, h$ is unbounded, $|g(m)| \geq \max (2, \sqrt{12 \delta})$ for some positive integer $m$ and

$$
|B(x, y) \Gamma(x+y)-g(x) h(y)| \leq \delta
$$

for all $x, y \in(0, \infty)$, where $B(x, y)$ is the beta function and $\Gamma(x)$ is the gamma function. Then

$$
B(x, y) g(x+y)=g(x) g(y)
$$

for all $x, y \in(0, \infty)$.

Corollary 3 Let $\delta>0$ and $a>1$ be given. Suppose that $f:(0, \infty) \rightarrow(0, \infty)$ is a function with $|f(m)| \geq \max \left\{2,(12 \delta)^{1 / 3}\right\}$ for some positive integer $m$ and

$$
\left|\frac{1}{a^{x y}} f(x+y)-f(x) f(y)\right| \leq \delta
$$

for all $x, y \in(0, \infty)$. Then

$$
f(x+y)=a^{x y} f(x) f(y)
$$

for all $x, y \in(0, \infty)$.

Proof Let $\beta(x, y)=\frac{1}{a^{x y}}$ for all $x, y \in(0, \infty)$. Then $\beta(x, y)=\beta(y, x)$ and $0<\beta(x, y)<1$. Also,

$$
\frac{\beta(x, y) \beta(z, x+y)}{\beta(x, y+z) \beta(y, z)}=\frac{a^{x(y+z)} a^{y z}}{a^{x y} a^{z(x+y)}}=1
$$


for all $x, y, z \in(0, \infty)$ and

$$
\prod_{i=1}^{n-1} \beta(i m, m)=\prod_{i=1}^{n-1} \frac{1}{a^{i m^{2}}} \rightarrow 0
$$

as $n \rightarrow \infty$. Also, $\beta(x, n)<\infty$ for fixed $x$. Thus, $\beta(x, y)$ is a beta-type function. By Theorem 1 with $\phi(x)=\delta$ and $s=m$, we complete the proof.

Corollary 4 Let $\delta>0$ and $k>1$ be given. Suppose that $f: R \rightarrow R$ is a function with $|f(m)| \geq$ $\max \left\{2,(12 \delta)^{1 / 3}\right\}$ for some positive integer $m$ such that

$$
\left|\frac{1}{k} f(x+y)-f(x) f(y)\right| \leq \delta
$$

for all $x, y \in R$. Then

$$
f(x+y)=k f(x) f(y)
$$

for all $x, y \in R$.

Proof By Theorem 1 with $\beta(x, y)=\frac{1}{k}, s=m$ and $\phi(x)=\delta$, we complete the proof.

4 Superstability of a gamma-beta type functional equation in the sense of Ger

Ger [9] suggested a new type of stability for the exponential equation of the following form:

$$
\left|\frac{f(x+y)}{f(x) f(y)}-1\right| \leq \delta
$$

In this section, the superstability problem in the sense of Ger for a gamma-beta type functional equation will be investigated.

Theorem 2 Let $\varphi: D \times D \rightarrow(0,1)$ be a function such that $\varphi\left(x, y_{n}\right) \rightarrow 0$ as $y_{n} \rightarrow \infty$ and let a beta-type function $\beta: D \times D \rightarrow(0, \infty)$ be given.

(a) Suppose that a function $f: D \rightarrow(0, \infty)$ satisfies

$$
\left|\frac{\beta(x, y) f(x+y)}{f(x) f(y)}-1\right| \leq \varphi(x, y)
$$

for all $(x, y) \in D \times D$. Then

$$
\beta(x, y) f(x+y)=f(x) f(y)
$$

for all $(x, y) \in D \times D$.

(b) Suppose that the inequality (4.1) holds and functions $f, g, h: D \rightarrow(0, \infty)$ satisfy

$$
\left|\frac{\beta(x, y) f(x+y)}{g(x) h(y)}-1\right| \leq \varphi(x, y)
$$


for all $(x, y) \in D \times D$. If $f(s)=g(s)$ for some $s \in D$, then

$$
\beta(x, y) g(x+y)=g(x) g(y)
$$

for all $(x, y) \in D \times D$.

(c) Suppose that the inequalities (4.1) and (4.2) hold. If $f(s)=h(s)$ for some $s \in D$ and $\varphi(x, y)=\varphi(y, x)$, then

$$
\beta(x, y) h(x+y)=h(x) h(y)
$$

for all $(x, y) \in D \times D$.

Proof (a) Choose a sequence $\left\{y_{n}\right\}$ in $D$ such that $y_{n} \rightarrow \infty$. For all $x, y, y_{n} \in D$, we have

$$
\begin{aligned}
\frac{\beta(x, y) f(x+y)}{f(x) f(y)}= & \frac{f(x+y) f\left(y_{n}\right)}{\beta\left(x+y, y_{n}\right) f\left(x+y+y_{n}\right)} \cdot \frac{\beta\left(x, y+y_{n}\right) f\left(x+y+y_{n}\right)}{f(x) f\left(y+y_{n}\right)} \\
& \cdot \frac{\beta\left(y, y_{n}\right) f\left(y+y_{n}\right)}{f(y) f\left(y_{n}\right)} \cdot \frac{\beta(x, y) \beta\left(x+y, y_{n}\right)}{\beta\left(x, y+y_{n}\right) \beta\left(y, y_{n}\right)} .
\end{aligned}
$$

By the condition (c) of a beta-type function $\beta$ and (4.1), we have

$$
\begin{aligned}
\lim _{y_{n}} \rightarrow \infty & \frac{1}{1+\varphi\left(x+y, y_{n}\right)}\left(1-\varphi\left(x, y+y_{n}\right)\right)\left(1-\varphi\left(y, y_{n}\right)\right) \\
& =1 \\
& \leq \frac{\beta(x, y) f(x+y)}{f(x) f(y)} \\
& \leq \lim _{y_{n} \rightarrow \infty} \frac{1}{1-\varphi\left(x+y, y_{n}\right)}\left(1+\varphi\left(x, y+y_{n}\right)\right)\left(1+\varphi\left(y, y_{n}\right)\right) \\
& =1
\end{aligned}
$$

for all $x, y \in D$. Thus, we complete the proof of (a).

(b) Choose a sequence $\left\{y_{n}\right\}$ in $D$ such that $y_{n} \rightarrow \infty$. For all $y, y_{n} \in D$, we have

$$
\frac{h\left(y+y_{n}\right)}{f\left(y+y_{n}\right)}=\frac{\beta\left(s, y+y_{n}\right) f(s+y+y n)}{f\left(y+y_{n}\right) f(s)} \cdot \frac{g(s) h\left(y+y_{n}\right)}{\beta\left(s, y+y_{n}\right) f\left(s+y+y_{n}\right)}
$$

and for all $x, y, y_{n} \in D$, we get

$$
\begin{aligned}
\frac{\beta(x, y) g(x+y)}{g(x) g(y)}= & \frac{g(x+y) h\left(y_{n}\right)}{\beta\left(x+y, y_{n}\right) f\left(x+y+y_{n}\right)} \cdot \frac{\beta\left(x, y+y_{n}\right) f\left(x+y+y_{n}\right)}{g(x) h\left(y+y_{n}\right)} \\
& \cdot \frac{h\left(y+y_{n}\right)}{f\left(y+y_{n}\right)} \cdot \frac{\beta\left(y, y_{n}\right) f\left(y+y_{n}\right)}{g(y) h\left(y_{n}\right)} \cdot \frac{\beta(x, y) \beta\left(x+y, y_{n}\right)}{\beta\left(x, y+y_{n}\right) \beta\left(y, y_{n}\right)} .
\end{aligned}
$$

By the condition (c) of a beta-type function $\beta$ and (4.2), we have

$$
\begin{aligned}
& \lim _{y_{n} \rightarrow \infty} \frac{1}{1+\varphi\left(x+y, y_{n}\right)}\left(1-\varphi\left(x, y+y_{n}\right)\right) \frac{1}{1+\varphi\left(s, y+y_{n}\right)}\left(1-\varphi\left(y, y_{n}\right)\right) \\
& \quad=1
\end{aligned}
$$




$$
\begin{aligned}
& \leq \frac{\beta(x, y) g(x+y)}{g(x) g(y)} \\
& \leq \lim _{y_{n} \rightarrow \infty} \frac{1}{1-\varphi\left(x+y, y_{n}\right)}\left(1+\varphi\left(x, y+y_{n}\right)\right) \frac{1}{1-\varphi\left(s, y+y_{n}\right)}\left(1+\varphi\left(y, y_{n}\right)\right) \\
& =1
\end{aligned}
$$

for all $x, y \in D$. Thus, we complete the proof of (b). Similarly, we obtain (c) from (b).

Remark 1 Consider the following inequalities: For all $(x, y) \in D \times D$,

$$
\frac{1}{1+\varphi(x, y)} \leq \frac{\beta(x, y) f(x+y)}{f(x) f(y)} \leq 1+\varphi(x, y)
$$

and

$$
\frac{1}{1+\varphi(x, y)} \leq \frac{\beta(x, y) f(x+y)}{g(x) h(y)} \leq 1+\varphi(x, y),
$$

where $\varphi: D \times D \rightarrow(0, \infty)$ is a function such that $\varphi\left(x, y_{n}\right) \rightarrow 0$ as $y_{n} \rightarrow \infty$. If we replace the inequality (4.1) by (4.3) and (4.2) by (4.3) respectively, then we have the same result as Theorem 2.

Corollary 5 Let $\varphi:(0, \infty) \times(0, \infty) \rightarrow(0,1)$ be a function such that $\varphi\left(x, y_{n}\right) \rightarrow 0$ as $y_{n} \rightarrow \infty$ and let the beta function $B$ and the gamma function $\Gamma$ be given. If functions $g, h:(0, \infty) \rightarrow(0, \infty)$ satisfy

$$
\left|\frac{B(x, y) \Gamma(x+y)}{g(x) h(y)}-1\right| \leq \varphi(x, y)
$$

for all $(x, y) \in(0, \infty) \times(0, \infty)$. If $g(1)=1$, then

$$
\beta(x, y) g(x+y)=g(x) g(y)
$$

for all $(x, y) \in(0, \infty) \times(0, \infty)$.

Corollary 6 Let $\varphi:(0, \infty) \times(0,1) \rightarrow(0, \infty)$ be a function such that $\varphi\left(x, y_{n}\right) \rightarrow 0$ as $y_{n} \rightarrow$ $\infty$ and let $a>1$ be given. If a function $f:(0, \infty) \rightarrow(0, \infty)$ satisfies

$$
\left|\frac{\frac{1}{a^{x y}} f(x+y)}{f(x) f(y)}-1\right| \leq \varphi(x, y)
$$

for all $(x, y) \in(0, \infty) \times(0, \infty)$, then

$$
\frac{1}{a^{x y}} f(x+y)=f(x) f(y)
$$

for all $(x, y) \in(0, \infty) \times(0, \infty)$.

Corollary 7 Let $\varphi:(0, \infty) \times(0, \infty) \rightarrow(0,1)$ be a function such that $\varphi\left(x, y_{n}\right) \rightarrow 0$ as $y_{n} \rightarrow$ $\infty$ and let $k>1$ be given. If a function $f:(0, \infty) \rightarrow(0, \infty)$ satisfies

$$
\left|\frac{\frac{1}{k} f(x+y)}{f(x) f(y)}-1\right| \leq \varphi(x, y)
$$


for all $(x, y) \in(0, \infty) \times(0, \infty)$, then

$$
\frac{1}{k} f(x+y)=f(x) f(y)
$$

for all $(x, y) \in(0, \infty) \times(0, \infty)$.

\section{Competing interests}

The author did not provide this information.

\section{Acknowledgements}

The author of this work was supported by the Basic Science Research Program through the National Research Foundation of Korea (NRF) funded by the Ministry of Education, Science and Technology (Grant No. 2012-0008382).

Received: 15 November 2012 Accepted: 14 December 2012 Published: 9 January 2013

\section{References}

1. Ulam, SM: Problems in Modern Mathematics. Proc. Chap. VI. Wiley, New York (1964)

2. Hyers, DH: On the stability of the linear functional equation. Proc. Natl. Acad. Sci. USA 27, $222-224$ (1941)

3. Rassias, ThM: The problem of S.M. Ulam for approximately multiplication mappings. J. Math. Anal. Appl. 246, 352-378 (2000)

4. Forti, GL: Hyers-Ulam stability of functional equations in several variables. Aequ. Math. 50, 146-190 (1995)

5. Baker, J, Lawrence, J, Zorzitto, F: The stability of the equation $f(x+y)=f(x)+f(y)$. Proc. Am. Math. Soc. 74, $242-246$ (1979)

6. Baker, J: The stability of the cosine equations. Proc. Am. Math. Soc. 80, 411-416 (1980)

7. Lee, YW: Superstability and stability of the Pexiderized multiplicative functional equation. Hindawi Pub. Corp. J. Inequal. Appl. 2010, 486325 (2010). doi:1155/2010/486325

8. Lee, YW, Kim, GH: Approximate gamma-beta type functions. Nonlinear Anal., Theory Methods Appl. 71, e1567-e1574 (2009)

9. Ger, R: Superstability is not natural. Rocznik Naukowo-Dydaktyczny WSP Krakkowie, Prace Mat. 159, 109-123 (1993)

doi:10.1186/1029-242X-2013-14

Cite this article as: Lee: Approximate pexiderized gamma-beta type functions. Journal of Inequalities and Applications 2013 2013:14

\section{Submit your manuscript to a SpringerOpen ${ }^{\ominus}$ journal and benefit from:}

- Convenient online submission

- Rigorous peer review

Immediate publication on acceptance

Open access: articles freely available online

- High visibility within the field

- Retaining the copyright to your article 